\title{
BIDIRECTIONAL REFLECTANCE OF FLAT, OPTICALLY THICK PARTICULATE LAYERS: AN EFFICIENT RADIATIVE TRANSFER SOLUTION AND APPLICATIONS TO SNOW AND SOIL SURFACES
}

\section{MICIAEL I. MISHCHENKO, "† JANTA M. DLUGACH EDGARD G. YANOVITSKIJ and NADIA T. ZAKHAROVA§}

$\uparrow$ NASA Goddard Institute for Space Studies, 2880 Broadway, New York, NY 10025, U.S.A. $\ddagger$ Laboratory for Radiative Transfer Theory, The Main Astronomical Observatory, Ukrainian National Academy of Sciences, Golosiiv, 252650 Kyiv-22, Ukraine. § Science Systems and Applications, Inc., 2880 Broadway, New York, NY 10025, U.S.A.

Abstract-We describe a simple and highly efficient and accurate radiative transfer technique for computing bidirectional reflectance of a macroscopically flat scattering layer composed of nonabsorbing or weakly absorbing, arbitrarily shaped, randomly oriented and randomly distributed particles. The layer is assumed to be homogeneous and optically semi-infinite, and the bidirectional reflection function (BRF) is found by a simple iterative solution of the Ambartsumian's nonlinear integral equation. As an exact solution of the radiative transfer equation, the reflection function thus obtained fully obeys the fundamental physical laws of energy conservation and reciprocity. Since this technique bypasses the computation of the internal radiation field, it is by far the fastest numerical approach available and can be used as an ideal input for Monte Carlo procedures calculating BRFs of scattering layers with macroscopically rough surfaces. Although the effects of packing density and coherent backscattering are currently neglected, they can also be incorporated. The FORTRAN implementation of the technique is available on the World Wide Web at http://www.giss.nasa.gov/ crmim/brf.html and can be applied to a wide range of remote sensing, engineering, and biophysical problems. We also examine the potential effect of ice crystal shape on the bidirectional reflectance of flat snow surfaces and the applicability of the HenyeyGreenstein phase function and the $\delta$-Eddington approximation in calculations for soil surfaces.

\footnotetext{
To whom all correspondence should be addressed. E-mail: crmim@giss.nasa.gov; fax: 2126785622.
} 


\section{INTRODUCTION}

Nany remote sensing, engineering, and biophysical applications rely on accurate knowledge of the bidirectional reflection function (BRF) of layers composed of discrete. randomly positioned scattering particles (e.g., Refs. 1-46). Theoretical computations of BRFs for plane-parallel particulate layers are usually reduced to solving the radiative transfer equation (RTE) using one of existing exact or approximate techniques. Since some semi-empirical approximate approaches such as the Hapke model $l^{13}$ are notorious for their low accuracy, crude violation of the energy conservation law, and ability to produce unphysical results, ${ }^{28,31}$ the use of numerically exact solutions of RTE has gained justified popularity. For example, the computation of BRFs for particulate layers with macroscopically flat surfaces in Refs. 5, 17, and 19-22 is based on the adding-doubling technique, ${ }^{47 .+8}$ while Refs. 9 and 10 employ the discrete ordinate method. ${ }^{49}$ BRF computations for layers with undulated (macroscopically rough) surfaces are more complicated and often may have to rely on time-consuming Monte Carlo procedures. This approach is especially inefficient for optically thick, weakly absorbing media (e.g., snow and desert surfaces at visible wavelengths) since a photon may undergo many internal scattering events before it exits the medium or is absorbed. However, particulate layers with undulated surfaces can often be represented as collections of locally flat tilted facets characterized by the BRF found from the traditional plane-parallel RTE. In this way the Monte Carlo procedure could be used only to evaluate the effects of surface shadowing and multiple surface reflections, thereby bypassing the time-consuming ray tracing inside the medium and providing a great saving of CPU time.

A further saving of computer resources can be achieved by using a more efficient technique for solving the plane-parallel RTE for a semi-infinite medium than the adding/doubling and discrete ordinate methods. Since many natural and artificial particulate 
layers can be considered optically semi-infinite and homogeneous, one can find the BRF directly by solving the Ambartsumian's nonlinear integral equation ${ }^{511}$ using a simple iterative technique. ${ }^{5.52}$ In this way, the computation of the internal radiation ficld is avoided (cf. Refs. 47-49) and the computer code becomes highly efficient and very accurate and compact. In the following sections, we discuss in detail numerical aspects and the computer implementation of this technique, examine the applicability of the Henyey-Greenstein phase function and the $\delta$ Eddington approximation in BRF and flux calculations for soil surfaces, and describe sample applications demonstrating the potential effect of ice crystal shape on the bidirectional reflectance of flat snow surfaces. The last section summarizes the results of the paper and outlines further potential improvements of the model.

\section{COMPUTATIONAL TECHNIQUE}

We assume that the scattering layer is optically semi-infinite, has a macroscopically flat surface, and is composed of randomly distributed and randomly oriented particles of arbitrary shape. For simplicity, we ignore polarization effects and use intensity as the only physical characteristic of light. This so-called scalar approximation is not necessarily good for Rayleigh scattering, ${ }^{53,54}$ but appears to be sufficiently accurate for particles with sizes comparable to and larger than the wavelength. ${ }^{55}$ To describe the geometry of light scattering, we use a right-handed spherical coordinate system with the $z$ axis directed along the outward normal to the surface (Fig. 1). The direction of light propagation is specified by the couple $(u, \varphi)$, where $u=-\cos \vartheta, \vartheta \in$ $\left[0^{\circ}, 180^{\circ}\right]$ is the zenith angle, and $\varphi \in\left[0^{\circ}, 360^{\circ}\right]$ is the azimuth angle. The azimuth angle is measured in the clock-wise direction when looking in the positive $z$ direction. Note that $u<0$ for upwelling radiation and $u>0$ for downwelling radiation. We also define $\mu=|u|$. The surface is 
illuminated by a beam of unpolarized light incident in the direction $\left(\mu_{n}, \varphi_{11}=0\right)$. The intensity of the reflected radiation is given by

$$
I(-\mu, \varphi)=\mu_{0} R\left(\mu, \mu_{0}, \varphi\right) F
$$

where $R\left(\mu, \mu_{1}, \varphi\right)$ is the bidirectional reflection function and $\pi F$ is the incident flux per unit area perpendicular to the incident beam. We ignore the effects of packing density, coherent backscattering, and shadow hiding (see Section 5) and find the reflection function as a numerically exact solution of the conventional radiative transfer equation. ${ }^{50,56}$ Specifically, we expand $R\left(\mu, \mu_{0}, \varphi\right)$ in a Fourier series in azimuth,

$$
R\left(\mu, \mu_{0}, \varphi\right)=R^{0}\left(\mu, \mu_{0}\right)+2 \sum_{m=1}^{m_{\text {max }}} R^{m}\left(\mu, \mu_{0}\right) \cos m \varphi
$$

and solve numerically the Ambartsumian's nonlinear integral equation ${ }^{50,51}$

$$
\begin{aligned}
\left(\mu+\mu_{0}\right) R^{m}\left(\mu, \mu_{0}\right)= & \frac{\varpi}{4} P^{m}\left(-\mu, \mu_{0}\right) \\
& +\frac{\varpi}{2} \mu_{0} \int_{0}^{1} P^{m}\left(\mu, \mu^{\prime}\right) R^{m}\left(\mu^{\prime}, \mu_{0}\right) \mathrm{d} \mu^{\prime} \\
& +\frac{\varpi}{2} \mu \int_{0}^{1} R^{m}\left(\mu, \mu^{\prime}\right) P^{m}\left(\mu^{\prime}, \mu_{0}\right) \mathrm{d} \mu^{\prime} \\
& +\varpi \mu \mu_{0} \int_{0}^{1} \int_{0}^{1} R^{m}\left(\mu, \mu^{\prime}\right) P^{m}\left(-\mu^{\prime}, \mu^{\prime \prime}\right) R^{m}\left(\mu^{\prime \prime}, \mu_{0}\right) \mathrm{d} \mu^{\prime} \mathrm{d} \mu^{\prime \prime},
\end{aligned}
$$

where $\varpi$ is the single scattering albedo and $P^{\prime \prime \prime}\left(\mu, \mu^{\prime}\right)$ are Fourier components of the phase function:

$$
P\left(\mu, \mu^{\prime}, \varphi-\varphi^{\prime}\right)=P^{0}\left(\mu, \mu^{\prime}\right)+2 \sum_{m=1}^{m_{\max }} P^{m}\left(\mu, \mu^{\prime}\right) \cos m\left(\varphi-\varphi^{\prime}\right)
$$


The upper summation limit in E: (2) is chosen such that the absolute numerical accuracy of the Fourier expansion of the reflection function is better than a small predefined number $\epsilon$ (e.g., $\epsilon=$ $\left.10^{-4}\right)$

The Fourier components of the phase function are given by

$$
P^{m}\left(\mu, \mu^{\prime}\right)=(-1)^{m} \sum_{s=m}^{s_{\max }} \alpha_{s} P_{m 0}^{s}(\mu) P_{m 0}^{s}\left(\mu^{\prime}\right)
$$

where $P_{m n}^{s}(x)$ are generalized spherical functions ${ }^{57,58}$ closely related to associate Legendre functions (Section 3.2) and $\alpha_{s}$ are expansion coefficients appearing in the standard expansion of the phase function $P(\Theta)$ in Legendre polynomials $P_{s}(x)=P_{00}^{s}(x)$ :

$$
P(\Theta)=\sum_{s=0}^{s_{\max }} \alpha_{s} P_{s}(\cos \Theta), \quad \alpha_{0} \equiv 1
$$

where $\Theta$ is the scattering angle and $s_{\max }$ is chosen such that all expansion coefficients with $s>s_{\max }$ are smaller than $0.1 \epsilon$. Note that $m_{\max } \leq s_{\max }$ and we assume the following standard normalization of the phase function:

$$
\frac{1}{2} \int_{0}^{\pi} P(\Theta) \sin \Theta \mathrm{d} \Theta=1
$$

If the expansion coefficients $\alpha_{s}$ are known, then one can easily compute the Fourier components of the phase function via Eq. (5) and finally solve Eq. (3) using the method of simple iterations.

It has been found that the method of simple iterations works very well for all $m>0$. Furthermore, convergence is reasonably fast for $m=0$ provided that the particles are absorbing $(\varpi<1)$. However, iterations converge very slowly or even may diverge for nonabsorbing or weakly absorbing particles $(1-\varpi<1) .^{51}$ It has been proved mathematically that this behavior is explained by the non-uniqueness of solutions of Eq. (3). ${ }^{59}$ To ameliorate this convergence 
problem, Dlugach and Yanovitskij ${ }^{51}$ suggested to modify the $R^{\prime \prime}\left(\mu, \mu_{0}\right)$ value after each iteration by enforcing the so-called Sobolev-van de Hulst retation

$$
i(-\mu)=2 \int_{0}^{1} R^{n \prime}\left(\mu, \mu_{0}\right) i\left(\mu_{0}\right) \mu_{0} \mathrm{~d} \mu_{0}
$$

The function $i(u)$ is the solution of the equation

$$
i(u)(1-k u)=\frac{\Phi}{2} \int_{-1}^{+1} i\left(u^{\prime}\right) P^{0}\left(u, u^{\prime}\right) \mathrm{d} u^{\prime},
$$

in which the so-called diffusion exponent $k$ is found by satisfying the normalization condition

$$
\frac{\varpi}{2} \int_{-1}^{+1} i(u) \mathrm{d} u=1
$$

After Eq. (3) is solved for each $m$, the evaluation of the Fourier series of Eq. (2) finalizes the process of computing the reflection function for any $\mu, \mu_{0}$, and $\varphi$. This function can then be used to calculate the reflected intensity for any directions of illumination and reflection and to find the plane, $A_{P}\left(\iota_{0}\right)$, and the spherical, $A_{s}$, albedos:

$$
\begin{aligned}
& A_{P}\left(\mu_{0}\right)=\frac{1}{\pi} \int_{0}^{1} \mathrm{~d} \mu \mu \int_{0}^{2 \pi} \mathrm{d} \varphi R\left(\mu, \mu_{0}, \varphi\right)=2 \int_{0}^{1} R^{0}\left(\mu, \mu_{0}\right) \mu \mathrm{d} \mu, \\
& A_{S}=2 \int_{0}^{1} A_{P}\left(\mu_{0}\right) \mu_{0} \mathrm{~d} \mu_{0} .
\end{aligned}
$$

Note that the BRF thus computed satisfies the fundamental principle of reciprocity:

$$
R\left(\mu, \mu_{0}, \varphi\right)=R\left(\mu_{0}, \mu, \varphi\right)
$$

Furthermore, since $i( \pm \mu) \equiv 1$ if $\varpi=1$, enforcing Eq. (8) ensures energy conservation for a semiinfinite nonabsorbing medium by rendering the plane and spherical albedos equal to 1 [cf. Eqs. (8), (11), and (12)]. 


\section{NUMERICAL ASPECTS AND COMPUTER CODES}

In this section we discuss numerical aspects and a FORTRAN implementation of the technique briefly outlined in the previous section. All computer procedures described are openly available on the World Wide Web at http://www.giss.nasa.gov/ crmim/brf.html.

\subsection{Legendre expansion of the phase function}

The widely used Henyey-Greenstein phase function and its Legendre expansion coefficients are given by the following simple formulas:

$$
\begin{aligned}
& P(\Theta)=\frac{1-g^{2}}{\left(1-2 g \cos \Theta+g^{2}\right)^{3 / 2}}, \quad g \in[-1,1], \\
& \alpha_{s}=(2 s+1) g^{s} .
\end{aligned}
$$

Note that

$$
g=<\cos \Theta>
$$

where

$$
<\cos \Theta>=\frac{1}{2} \int_{-1}^{+1} P(\Theta) \cos \Theta \mathrm{d}(\cos \Theta)=\frac{1}{3} \alpha_{1}
$$

is the asymmetry parameter of the phase function. Similarly, for the often used double-peaked Henyey-Greenstein phase function ${ }^{60}$

$$
P(\Theta)=f \frac{1-g_{1}^{2}}{\left(1-2 g_{1} \cos \Theta+g_{1}^{2}\right)^{3 / 2}}+(1-f) \frac{1-g_{2}^{2}}{\left(1-2 g_{2} \cos \Theta+g_{2}^{2}\right)^{3 / 2}}
$$

with a positive $g_{1}$ and a negative $g_{2}$, the Legendre expansion coefficients are given by

$$
\alpha_{s}=f \alpha_{s 1}+(1-f) \alpha_{s 2}
$$

where $\alpha_{s 1}$ and $\alpha_{s 2}^{s}$ are given by Eq. (15) with $g=g_{1}$ and $g=g_{2}$, respectively. 
The code for computing the expansion enefficients for polydisperse, homogeneous spherical particles is based on the standard Lorenz-Mie theory and the approach described in Refs. 61 and 62. The code allows one to select one of the following five size distributions:

- the modified gamma distribution

$$
n(r)=\text { const } \times r^{\alpha} \exp \left(-\frac{\alpha r^{\gamma}}{\gamma r_{c}^{\gamma}}\right):
$$

- the log normal distribution

$$
n(r)=\text { const } \times r^{-1} \exp \left(-\frac{\left(\ln r-\ln r_{g}\right)^{2}}{2 \ln ^{2} \sigma_{g}}\right)
$$

- the power law distribution

$$
n(r)=\left\{\begin{array}{l}
\text { const } \times r^{-3}, r_{1} \leq r \leq r_{2}, \\
0, \text { otherwise }
\end{array}\right.
$$

- the gamma distribution

$$
n(r)=\text { const } \times r^{(1-3 b) / b} \exp \left(-\frac{r}{a b}\right), \quad b \in(0,0.5)
$$

- the modified power law distribution

$$
n(r)=\left\{\begin{array}{l}
\text { const, } 0 \leq r \leq r_{1}, \\
\text { const } \times\left(r / r_{1}\right)^{\alpha}, r_{1} \leq r \leq r_{2}, \\
0, r_{2}<r .
\end{array}\right.
$$

The constant for each size distribution is chosen such that the size distribution satisfies the standard normalization

$$
\int_{r_{\min }}^{r_{\operatorname{mix}}} n(r) \mathrm{d} r=1
$$


Mathematically, particle radii in the modified gamma, log normal, and gamma distributions may extend to infinity. However, a tinite $r_{\text {max }}$ must be chosen in actual computer calculations. There are two practical interpretations of a truncated size distribution. First, $r_{\text {max }}$ can be increased until the scattering characteristics converge within some numerical accuracy (note that convergent $r_{\text {max }}$ values for the modified gamma and log normal distributions can be unrealistically large for small $\gamma$ or large $\sigma_{g}{ }^{63}$ ). In this case the converged truncated size distribution is numerically equivalent to the distribution with $r_{\max }=\infty$. Second, a truncated distribution can be considered a specific distribution with scattering characteristics different from those for the distribution with $r_{\max }=\infty$. Similar considerations apply to the parameter $r_{\min }$ whose mathematical value for the modified gamma, log normal, and gamma distributions is zero, but in practice can be any number smaller than $r_{\max }$. Note that for the gamma distribution with $r_{\min }=0$ and $r_{\max }=\infty, a$ and $b$ coincide with the effective radius $r_{\text {eff }}$ and effective variance $v_{\text {eff }}$, respectively, as defined by Hansen and Travis. ${ }^{47}$

The numerical integration of scattering characteristics over a size distribution is achieved by subdividing the entire interval $\left[r_{\min }, r_{\max }\right]$ of particle radii into a number $n$ of equal subintervals and applying a Gaussian quadrature formula with $k$ division points to each subinterval. Note that $n$ and/or $k$ should be increased until the required numerical accuracy is reached.

An efficient technique for computing the Legendre expansion coefficients for polydispersions of randomly oriented, homogeneous, rotationally symmetric nonspherical particles is described in detail in Ref. 64. This technique is based on the $T$-matrix approach ${ }^{65}$ and an analytical method for averaging scattering characteristics over particle orientations. ${ }^{66}$ 
The computation of the Legendre expansion coefficients for phase functions obtained with other numerical methods or measured experimentally is based on the numerical evaluation of the integral

$$
\alpha_{s}=\frac{2 s+1}{2} \int_{0}^{\pi} \mathrm{d} \Theta \sin \Theta P(\Theta) P_{s}(\cos \Theta),
$$

which is a direct consequence of $\mathrm{Eq}$. (6) and the orthogonality relation for Legendre polynomials. The integral is replaced by a Gaussian quadrature and an interpolation procedure is employed to find the phase function at Gaussian division points using the table of pre-computed or measured phase function values. The Legendre polynomials are computed using the recurrence relation and the initial conditions given by Eqs. (27) and (28) below with $m=0$. We have found that spline interpolation usually provides quite acceptable results with the exception of phase functions having very sharp features, ${ }^{67}$ such as the phase function for hexagonal ice crystals. The presence of the strong $22^{\circ}$ and $46^{\circ}$ halos in this latter case ${ }^{68}$ necessitates the use of simple linear interpolation. Furthermore, the $\delta$-function transmission peak in the ray tracing phase function for hexagonal ice crystals must be convolved with the Fraunhofer pattern, as described in Ref. 69.

\subsection{Fourier components of the phase function}

The right-hand side of Eq. (5) is often written in terms of associated Legendre functions $P_{s}^{m}(x)=(-\mathrm{i})^{m}[(s+m) ! /(s-m) !]^{1 / 2} P_{0 m}^{s}(x), \quad \mathrm{i}=\sqrt{-1}$, rather than generalized spherical functions.

It is well known, however, that the numerical computation of associated Legendre functions with large $m$ and $s$ is unstable and leads to overflows. ${ }^{70}$ On the other hand, the computation of the generalized spherical functions via the upward recurrence relation ${ }^{57,58}$ 


$$
\sqrt{(s+1)^{2}-m^{2}} P_{m+1}^{r+1}(x)=(2 s+1) x P_{m(1)}^{s}(x)-\sqrt{s^{2}-m^{2}} P_{m 1)}^{s-1}(x)
$$

and initial conditions

$$
P_{m 0}^{m-1}(x)=0 . \quad P_{m 0}^{m}(x)=(2 \mathrm{i})^{-m} \sqrt{\frac{(2 m) !}{m ! m !}}\left(1-x^{2}\right)^{m / 2}
$$

is numerically stable and efficient. Furthermore, the concept of generalized spherical functions naturally appears in the theory of polarized radiative transfer, ${ }^{5,58}$ the Lorenz-Mie theory, ${ }^{62}$ and the $T$-matrix method ${ }^{66}$ and provides a natural and appealing link between these theories.

\subsection{Iterative solution of the Ambartsumian's equation}

By using a quadrature formula on the interval $\mu \in[0,1]$ with $n$ division points $\mu_{p}$ and weights $w_{p}$, we convert integral equation (3) into a system of $n \times n$ nonlinear algebraic equations

$$
\begin{aligned}
\left(\mu_{p}+\mu_{q}\right) R^{m}\left(\mu_{p}, \mu_{q}\right)= & \frac{\varpi}{4} P^{m}\left(-\mu_{p}, \mu_{q}\right) \\
& +\frac{\varpi}{2} \mu_{q} \sum_{s=1}^{n} w_{s} P^{m}\left(\mu_{p}, \mu_{s}\right) R^{m}\left(\mu_{s}, \mu_{q}\right) \\
& +\frac{\varpi}{2} \mu_{p} \sum_{s=1}^{n} w_{s} R^{m}\left(\mu_{p}, \mu_{s}\right) P^{m}\left(\mu_{s}, \mu_{q}\right) \\
& +\varpi \mu_{p} \mu_{q} \sum_{s=1}^{n} \sum_{s^{\prime}=1}^{n} w_{s} w_{s^{\prime}} R^{m}\left(\mu_{p}, \mu_{s}\right) P^{m}\left(-\mu_{s}, \mu_{s^{\prime}}\right) R^{m}\left(\mu_{s^{\prime}}, \mu_{q}\right)
\end{aligned}
$$

for the unknowns $R^{m}\left(\mu_{p}, \mu_{q}\right), p, q=1, \ldots, n$. This system is solved by simple iterations using

$$
R_{[0]}^{m}\left(\mu_{p}, \mu_{q}\right)=\frac{\varpi}{4\left(\mu_{p}+\mu_{q}\right)} P^{m}\left(-\mu_{p}, \mu_{q}\right)
$$

as the initial approximation. The iterations converge very fast for $m>0$ as well as for $m=0$ and $\varpi<0.8$. However, the convergence rate becomes very slow when $m=0$ and $1-\varpi<$ 
1. To accelerate convergence, we use a procedure similar to those developed in Retis. 51 and 52 and based on the Sobolev-van de Hulst relation. Specifically, after each iteration. we compute the quantities [cf. Eq. (8)]

$$
\Delta_{[\jmath]}\left(\mu_{p}\right)=i\left(-\mu_{p}\right)-2 \sum_{q=1}^{n} w_{q} R_{[\jmath\}}^{0}\left(\mu_{p}, \mu_{q}\right) \mu_{q} i\left(\mu_{q}\right)
$$

where $j$ numbers iterations. We then improve $R_{\mathrm{U} / 1}^{0}\left(\mu_{\rho}, \mu_{4}\right)$ by replacing it with

$$
R_{[j]}^{0}\left(\mu_{p}, \mu_{q}\right)+\kappa\left[\Delta_{[\jmath]}\left(\mu_{p}\right) i\left(\mu_{q}\right)+i\left(\mu_{p}\right) \Delta_{[J]}\left(\mu_{q}\right)\right]
$$

where $\mathrm{K}$ is an appropriately chosen constant. This improved approximation is substituted in Eq. (31) to compute a new set of quantities $\Delta_{[j]}\left(\mu_{p}\right)$, which are used again to further improve $R_{[j]}^{0}\left(\mu_{p}, \mu_{4}\right)$ via Eq. (32), and this procedure is repeated until

$$
\max _{p=1, \ldots, n}\left|\Delta_{[j]}\left(\mu_{p}\right)\right|<0.1 \epsilon
$$

where $\epsilon$ is the predefined absolute accuracy of computations. The improved $j$ th iteration $R_{[j]}^{0}\left(\mu_{p}, \mu_{q}\right)$ is then substituted in the right-hand side of Eq. (29) to obtain the $(j+1)$ th approximation $R_{[j+1]}^{0}\left(\mu_{p}, \mu_{q}\right)$, which is again improved using Eqs. (31) and (32), and this entire process is repeated until

$$
\max _{p, q=1, \ldots, n}\left|R_{[j+1]}^{0}\left(\mu_{p}, \mu_{q}\right)-R_{[j]}^{0}\left(\mu_{p}, \mu_{q}\right)\right|<\epsilon
$$

de Rooij ${ }^{52}$ suggested to use the same value $k=0.5$ in all cases. We have found, however, that this value may cause divergence when $\omega<0.995$ and that $\kappa$ should be single-scattering-albedo specific. After having performed many numerical experiments, we have chosen the following $\kappa$ values: 


$$
K=\left\{\begin{array}{l}
0.5 \text { for } \pi \geq 0.995 \\
0.1 \text { for } 0.95 \leq \pi<0.995 \\
0.05 \text { for } 0.8 \leq \pi<0.95
\end{array}\right.
$$

The use of reciprocity [Eq. (13)] reduces the number of unknowns in Eq. (29) by a factor of $2 N /(N+1)$ and provides a significant saving of computer resources.

This numerical procedure renders only reflection function values $R\left(\mu_{p}, \mu_{q}, \varphi\right)$ at the division points of the quadrature formula. BRF values for $\mu$ and $\mu_{0}$ not coinciding with one of the quadrature nodes must be found by numerical interpolation/extrapolation, which may result in lower accuracy than for the BRF values at the quadrature nodes. Therefore, the number of quadrature division points $n$ should be increased until the desired numerical accuracy for all required BRF values is achieved. The accuracy can be significantly improved and $n$ can be decreased by using the separation of the first-order scattering procedure (Section 3.7).

\subsection{Computation of $i(\mu)$}

Taking into account the normalization

$$
\frac{1}{2} \int_{-1}^{+1} P^{0}\left(u, u^{\prime}\right) \mathrm{d} u^{\prime}=1
$$

we derive from Eqs. (9) and (10)

$$
k=\frac{2(1-\varpi)}{\varpi \int_{-1}^{+1} u i(u) \mathrm{d} u} .
$$

Replacing the integrals in Eqs. (9), (10), and (37) by respective quadrature sums, we obtain

$$
i\left( \pm \mu_{p}\right)=\frac{\varpi}{2\left(1 \mp k \mu_{p}\right)} \sum_{q=1}^{n} w_{q}\left[i\left(\mu_{q}\right) P^{0}\left( \pm \mu_{p}, \mu_{q}\right)+i\left(-\mu_{q}\right) P^{0}\left( \pm \mu_{p},-\mu_{q}\right)\right],
$$




$$
\begin{aligned}
& \frac{\pi}{2} \sum_{p=1}^{n} w_{p}\left[i\left(\mu_{p}\right)+i\left(-\mu_{p}\right)\right]=1, \\
& k=\frac{2(1-\varpi)}{\varpi \sum_{p=1}^{n} w_{p} \mu_{p}\left[i\left(\mu_{p}\right)-i\left(-\mu_{p}\right)\right]} .
\end{aligned}
$$

Substituting $k=\sqrt{1-\bar{\sigma}}, i\left(\mu_{p}\right)=2$, and $i\left(-\mu_{p}\right)=1 / 2$ as the initial approximation, we calculate the right-hand side of Eq. (38) to obtain the next approximation for $i\left(\mu_{p}\right)$ and $i\left(-\mu_{p}\right)$. Since this approximation may not satisfy the normalization of Eq. (39), we improve $i\left(\mu_{p}\right)$ and $i\left(-\mu_{p}\right)$ by dividing them by

$$
\frac{\varpi}{2} \sum_{p=1}^{n} w_{p}\left[i\left(\mu_{p}\right)+i\left(-\mu_{p}\right)\right]
$$

This improved approximation satisfies Eq. (39) and is used to compute the next approximation for $k$ via Eq. (40). The new $k, i\left(\mu_{p}\right)$, and $i\left(-\mu_{p}\right)$ values are substituted in the right-hand side of Eq. (38) to obtain the next approximation for $i\left(\mu_{p}\right)$ and $i\left(-\mu_{p}\right)$, and so on. The process is continued until $i\left(\mu_{p}\right)$ and $i\left(-\mu_{p}\right)$ converge within $0.1 \epsilon$. Note that this scheme is different from that described in Ref. 51. Dlugach and Yanovitskij ${ }^{51}$ compute $i( \pm \mu)$ using the expansion coefficients $\alpha^{s}$ and a method of continued fractions. We have found, however, that the use of the expansion coefficients of the original phase function to compute $i( \pm \mu)$ conflicts with the use of the renormalized phase function (Section 3.6 below) in Eq. (29) and may lead to divergence of the iterative solution of Eq. (29) for highly anisotropic phase functions. Our new procedure for computing $i( \pm \mu)$ uses the already renormalized phase function and produces numerically stable and convergent results. 


\subsection{Vumerical incegration}

The Gauss quadrature formula (e.g., Ref. 71) has the highest algebraic degree of precision (i.e., a formula with $n$ nodes is exact for all polynomials of degree $2 n-1$ and lower) and is traditionally used in radiative transfer calculations to numerically evaluate integrals on the interval [0.1] (e.g.. Refs. 72-7ł). A significant disadvantage of this quadrature is that the largest node is always smaller than 1 , and if BRF values for normal incidence and/or reflection are required, then one must use an extrapolation procedure. Unfortunately, extrapolation often produces poor numerical accuracy (e.g., see discussion on pages 210 and 211 of Ref. 52) and necessitates the use of the Gaussian quadrature formula with a large number of nodes. We have found that a more efficient approach is to use the so-called Markov quadrature formula (Chapter 9.2 of Ref. 71 ) with one predefined node at $\mu=1$. This formula still has the highest possible algebraic precision and is exact for all polynomials of degree $2 n-2$ and lower. Furthermore, it allows one to avoid the use of the extrapolation procedure or so-called extra points (Ref. 47 and L. D. Travis, personal communication). Multiple numerical tests have shown that the Gaussian and Markov quadratures with a number of nodes $n$ larger than about 10 have essentially the same numerical accuracy for intermediate $\mu$ and $\mu_{0}$ values, whereas the Markov quadrature produces much better accuracy for $\mu$ and/or $\mu_{0}$ equal to 1 . Since the CPU time consumption in solving Eq. (29) is proportional to $n^{3}$, the use of the Markov quadrature with a reduced number of nodes results in a significant saving of computer resources. We have developed a simple, efficient, and highly accurate FORTRAN procedure for computing the nodes and weights of the Markov quadrature formula with an arbitrary $n$. Table 1 exemplifies the performance of the procedure and lists the nodes and weights of the Markov quadrature with $n=30$. 
The direct application of a quidrature formula to the integration $\mu$-interval $[0,1]$ is a standard approach in the radiative transfer theory (e.g., Refs. 47-49, 51, 55, 61, 72-74). However. it provides poor sampling of zenith angles close to $0^{\circ}$ and, as multiple numerical tests have shown, causes a very slow convergence of $R^{0}(1,1)$ with increasing $n$ for particles large compared to the wavelength. This happens even when the Markor quadrature is used and no extrapolation is involved or when the Gaussian quadrature is used along with an extra point at $\mu=\mu_{0}=1$. On the other hand, convergence with increasing $n$ is fast for $\mu<1$ and $\mu_{0}<1$. We have found that a very efficient way of avoiding excessive $n$ values in radiative transfer computations is to apply the Gaussian quadrature to the interval $[0, \pi / 2]$ of zenith angle values. Since

$$
\int_{0}^{1} f(\mu) \mathrm{d} \mu=\int_{0}^{\pi / 2} f(\cos \vartheta) \sin \vartheta \mathrm{d} \vartheta
$$

we easily derive the following expressions for the respective division points and weights in Eq. (29):

$$
\mu_{p}=\cos \left(\frac{\pi}{4} X_{p}+\frac{\pi}{4}\right), w_{p}=W_{p} \sin \left(\frac{\pi}{4} X_{p}+\frac{\pi}{4}\right), \quad p=1, \ldots, n,
$$

where $X_{p}$ and $W_{p}$ are Gaussian nodes and weights, respectively, on the interval $[-1,+1]$. These division points provide a much better sampling of zenith angles close to $0^{\circ}$ and a much higher convergence rate for $R^{0}(1,1)$ with increasing $n$ than the Gaussian or the Markov quadrature formula applied to the interval $[0,1]$ of $\mu$ values. 


\subsection{Renormalization of the phase function}

Although analytically the zeroth Fourier component of the phase function must be normalized according to Eq. (36), the numerical evaluation of the left-hand side of Eq. (36) usually produces $\mu$-dependent numbers not equal to 1 :

$$
\frac{1}{2} \sum_{q=1}^{n} w_{q}\left[P^{0}\left(\mu_{p}, \mu_{q}\right)+P^{0}\left(\mu_{p} \cdot-\mu_{q}\right)\right]=\delta_{p} \neq 1 .
$$

This results in a deviation of the "numerical" single scattering albedo from its actual value and, for nonabsorbing or weakly absorbing media, can lead to an efficient "photon gain" or "photon loss." A direct adverse consequence is a serious violation of energy conservation and poor numerical accuracy. Hansen ${ }^{55}$ developed a so-called renormalization procedure, which numerically enforces the normalization of Eq. (36) by slightly modifying the $P^{0}\left(\mu_{p}, \mu_{q}\right)$ values.

We have found that the renormalization procedure of Ref. 55 produces accurate BRFs in most cases, but not always. Therefore, we have developed an alternative renormalization procedure, which is simpler than that of Ref. 55 and appears to be more stable. Specifically, we multiply the quantities $P^{0}\left(\mu_{p}, \mu_{p}\right), p=1, \ldots, n$ by the correction factors

$$
\varepsilon_{p}=1+\frac{2-2 \delta_{p}}{w_{p} P^{0}\left(\mu_{p}, \mu_{p}\right)}, \quad p=1, \ldots, n
$$

This correction makes the left-hand side of Eq. (44) equal to 1 for any $p$ and is applied to higher Fourier components of the phase function as well. Since it affects only the forward-scattering values of the phase function, it has negligible effect on the bidirectional reflection function while numerically ensuring energy conservation. 


\section{3. . Separation of the first-order-scattering contribution to the reflection function}

For large scattering particles with highly variable phase functions and for $\mu$ and $\mu_{0}$ significantly smaller than 1, one may need very many Fourier terms in Eq. (2) in order to accurately represent the reflection function. On the other hand, it is also known that with $\mu$ and $\mu_{1)}$ approaching zero the only significant contribution to the reflection function comes from photons scattered only once. ${ }^{56}$ This suggests the idea of subtracting the first-order-scattering contribution from all Fourier components of the reflection function, thereby greatly reducing the number of numerically significant Fourier components, evaluating the right-hand side of Eq. (2), interpolating (if necessary) this slowly varying high-order-scattering part of the reflection

function, and finally adding the exact single-scattering contribution. ${ }^{75}$ The latter contribution can be easily computed analytically for the scattering angle $\Theta$ corresponding to a specific combination of $\mu, \mu_{0}$, and $\varphi$ values and given by

$$
\cos \Theta=-\mu \mu_{0}+\sqrt{1-\mu^{2}} \sqrt{1-\mu_{0}^{2}} \cos \varphi .
$$

In other words, the total reflection function is represented in the form

$$
R\left(\mu, \mu_{0}, \varphi\right)=R_{l}\left(\mu, \mu_{0}, \varphi\right)+\sum_{m=0}^{m_{1}}\left(2-\delta_{m 0}\right)\left[R^{m}\left(\mu, \mu_{0}\right)-R_{1}^{m}\left(\mu, \mu_{0}\right)\right] \cos m \varphi,
$$

where $\delta_{m m^{\prime}}$ is the Kronecker delta,

$$
\begin{aligned}
& R_{1}\left(\mu, \mu_{0}, \varphi\right)=\frac{\varpi}{4\left(\mu+\mu_{0}\right)} P(\Theta), \\
& R_{1}^{m}\left(\mu, \mu_{0}\right)=\frac{\varpi}{4\left(\mu+\mu_{0}\right)} P^{m}\left(-\mu, \mu_{0}\right),
\end{aligned}
$$


and $m_{1} \ll m_{\max }$. $^{+8}$ The term in square brackets on the right-hand side of Ec. (47) is a smooth function of $\mu$ and $\mu_{0}$ and can be accurately interpolated even when the number of quadrature nodes is relatively small, while $P(\Theta)$ is computed via Eqs. (6) and (46).

\section{COMPUTATIONS AND DISCUSSION}

\section{t.1. Soil surfaces}

Table 2 lists parameters of four soil particle models used in the computations described below. We assume the standard gamma size distribution of Eq. (23) with an effective radius of $a=r_{\mathrm{eff}}=10 \mu \mathrm{m}$ and an effective variance of $b=v_{\mathrm{eff}}=0.1$. This effective radius is typical of soil particles (e.g.. Ref. 76). The four values of the refractive index $m=m_{r}+i m_{t}$ are also typical of soil particles at the visible wavelength $\lambda=0.63 \mu \mathrm{m}$ considered. ${ }^{76}$ The single scattering properties of the four soil particle models were computed assuming the spherical particle shape and using the Lorenz-Mie theory. (It should be noted, however, that the Lorenz-Mie theory does not necessarily provide the best representation of soil particle phase functions. ${ }^{77-80}$ ) Table 2 gives the respective values of the single-scattering albedo $\varpi$, asymmetry parameter of the phase function $<\cos \Theta>$, the number of terms in the Legendre decomposition of the phase function $s_{\max }$ [Eq. (6)], and the spherical albedo $A_{S}$. Note the significant decrease of $\varpi$ and increase of $<\cos \Theta>$ with increasing imaginary part of the refractive index. The solid curves in the upper panel of Fig. 2 show the respective Lorenz-Mie phase functions, while the dotted curves show the asymmetry-parameter-equivalent Henyey-Greenstein phase functions [Eq. (14)].

Table 2 also gives spherical albedo values computed using the equivalent HenyeyGreenstein phase function, $A_{S}(\mathrm{HG})$, and the simple approximate formula 


$$
A_{S}(\mathrm{H} H \mathrm{H})=(1-s) /(1+s)
$$

derived by Hovenicr and Hage, ${ }^{81}$ where

$$
s=\sqrt{\frac{1-\omega}{1-\pi<\cos \Theta>}}
$$

is the so-called similarity parameter. ${ }^{56}$ It is seen that the $A_{S}(\mathrm{HG})$ values are quite close to the exact ones, while the Hovenier and Hage approximation provides somewhat lower accuracy.

Solid curves in the upper panel of Fig. 3 depict the plane albedo $A_{P}$ as a function of the cosine of the illumination zenith angle $\mu_{0}$. Note that $A_{p}$ is determined only by the Oth component of the reflection function via Eq. (11) and, as a consequence, the computation of the upper panel of Fig. 3 using 50 quadrature division points took less than 2 sec of CPU time on an IBM RISC model 397 workstation. We also computed the plane albedo using the asymmetryparameter-equivalent Henyey-Greenstein phase functions and the $\delta$-Eddington approximation. ${ }^{82}$ The ratios of these approximate plane albedo values relative to the exact ones are shown by the dotted and solid curves, respectively, in the bottom panel of Fig. 3. Not surprisingly, plane albedos decrease with increasing the imaginary part of the refractive index and, thus, decreasing the single-scattering albedo. Both the $\delta$-Eddington approximation and the asymmetry-parameterequivalent Henyey-Greenstein phase function produce significant errors, especially for grazing illumination. Using the asymmetry-parameter-equivalent Henyey-Greenstein phase function overestimates the plane albedo for small $\mu_{0}$ and underestimates it for $\mu_{0}$ close to 1 , which is naturally explained by the scattering-angle pattern of the phase function differences (upper panel of Fig. 2). The errors increase significantly with increasing absorption. This can be explained by the increasing contribution of photons scattered only once and by the large differences in the 
single-scattering phase functions. The much better accuracy of the $A_{5}(\mathrm{HG})$ values in Table 2 can be explained by cancellation of the plane albedo errors after integrating over $\mu_{0}$ in Eq. (12).

Figure 4 shows the angular distribution of the retlected intensity computed for soil particle model 1 using the exact Lorenz-Mie phase function and its Henyey-Greenstein counterpart and assuming $F=1$ in Eq. (1). The computations for the exact phase function used 100 quadrature nodes and took about 25 min of CPU time including the Lorenz-Mie computation of the Legendre expansion coefficients, the solution of Eq. (3), and interpolation. The computations for the equivalent Henyey-Greenstein phase function used 50 quadrature nodes and took less than $3 \mathrm{~min}$.

Two obvious features of the reflected intensity distributions shown in the left column are the backscattering enhancement $\left(\mu=\mu_{0}, \varphi=180^{\circ}\right)$ caused by the glory in the Lorenz-Mie phase function (upper panel of Fig. 2) and the strong near-forward scattering for the cases of grazing and near-grazing incidence caused by the phase function peak at small scattering angles. The reflectance patterns for the equivalent Henyey-Greenstein phase function exhibit only the second feature, which is explained by the absence of the backscattering phase function peak similar to the glory. The right column of Fig. 4 shows that BRF errors caused by the use of the equivalent Henyey-Greenstein phase function can be very large and can exceed a factor of 20 at backscattering geometries and a factor of 3 at near-forward-scattering geometries. These errors can be unequivocally attributed to the phase function differences. Thus, Fig. 4 makes a strong case against using approximate phase functions in BRF computations for semi-infinite particulate media. 


\subsection{Sinow surfacis}

In this section we describe BRF computations for three snow particle models summarized in Table 3. The assumed wavelength is $\lambda=0.65 \mu \mathrm{m}$. Model 1 particles have highly irregular, random-fractal shapes introduced in Ref. 83; model 2 particles are homogeneous ice spheres; and model 3 particles are regular hexagonal ice crystals with a length-to-diameter ratio of 2 . The nonspherical model 1 and 3 particles are randomly oriented in three-dimensional space. For all three models we used the same power law distribution of radii or projected-area-equivalentsphere radii [Eq. (22)] with an effective radius of $50 \mu \mathrm{m}$ and an effective variance of 0.2 . The respective phase functions were computed using the ray tracing technique ${ }^{83}$ coupled with the Kirchhoff approximation ${ }^{69}$ for models 1 and 3 and the Lorenz-Mie theory for model 2. They are shown in the lower panel of Fig. 2 and exhibit large differences exceeding an order of magnitude at some scattering angles. The resulting differences in the asymmetry parameter are also significant (Table 3). As discussed in Ref. 84, the phase functions of models 1 and 3 may represent limiting cases of highly distorted and pristine ice crystals, respectively. Since water ice is essentially nonabsorbing at visible wavelengths, the single-scattering, plane, and spherical albedos for all three models are equal to unity.

Figure 5 shows the reflected intensities for the three snow particle models, while Figure 6 depicts the ratios $2 / 1,3 / 1$, and $3 / 2$ of intensities for the respective models. It is obvious that the shape of the scattering particles has a profound effect on the reflectance of flat snow surfaces. Although radiance differences between the different models are relatively small at nearly normal incidence $\left(\mu_{0}=0.9\right.$ ), they significantly increase with decreasing $\mu_{0}$ and result in intensity ratios smaller than 0.2 and larger than 3 (cf. Ref. 84). This is a direct consequence of the increasing relative contribution of the first-order scattering to the reflection function and the large phase 
function differences. Hexagonal snow crystals (model 3) produce the most structured radiance lick dominated by the backscattering peak and the primary $\left(22^{\circ}\right)$ and secondary $\left(46^{\circ}\right)$ halos (two lower panels in the right column of Fig. 5). These features clearly show up in the $3 / 1$ and $3 / 2$ intensity ratios (Fig. 6). The spherical ice particles produce a noticeable enhancement of intensity caused by the rainbow. This feature is especially well seen in the $2 / 1$ ratio. The radiance field produced by the featureless phase function of irregular snow crystals (model 1 ) is by far the least structured (left column of Fig. 5). These results emphasize the importance of accurate treatment of single-scattering phase functions for realistic snow grain models.

\section{CONCLUSIONS}

We have described in detail an efficient technique for computing bidirectional reflectance of semi-infinite discrete random media based on an exact numerical solution of the radiative transfer equation. This technique results in a very compact and fast computer code and produces BRFs which fully comply with reciprocity and energy conservation. The high efficiency and accuracy of the technique make far less tempting the use of approximations such as the $\delta$ Eddington approximation, the asymmetry-parameter-equivalent Henyey-Greenstein phase function, and the truncation of the phase function ${ }^{73}$ and provide an ideal tool for testing the accuracy of approximate approaches. ${ }^{81,85}$ Our sample computations for flat soil and snow surfaces have clearly demonstrated the limited applicability of approximate treatments of the single-scattering phase function in BRF modeling.

Since we considered only nonabsorbing or weakly absorbing media, we ignored the opposition effect caused by so-called shadow hiding.' Other factors ignored in the model are the effects of polarization, ${ }^{53-55}$ packing density,,$^{35,41,86-91}$ and coherent backscattering..$^{29,30,32,38,43,92}$ 
However, they can be incorporated in a rather straightforward manner, as described, e.g., in Refs. $26-28,52$, and $93-95$. This is the subject of our current research. An equally challenging problem of macroscopic surface roughness ${ }^{8,12,19,24,16,96}$ can be addressed by convolving BRFs computed as described in this paper with a Monte Carlo procedure handling multiple surface reflections and surface shadowing. As pointed out in the introduction, this approach avoids time-consuming ray tracing inside a nonabsorbing or weakly absorbing. optically semi-infinite medium and provides a great saving of computer resources. The ultimate challenge is to take into account the effects of the discontinuous nature of light scattering in densely packed discrete random media, ${ }^{31,35,97}$ but this requires the development of a much more sophisticated approach.

Acknowledgements-We thank Brian Cairns, Joop Hovenier, and Andrew Lacis for careful reading of the manuscript and useful comments and Lilly Del Valle for help with graphics. This research was sponsored by the NASA Radiation Science Program and NASA Global Aerosol Climatology Project managed by Robert Curran. 


\section{REFERENCES}

I. Bowell, E., Hapke, B., Domingue, D., Lumme, K., Peltoniemi, J. and Harris, A. W., Application of photometric models to asteroids. In Asteroids II, R. P. Binzel, T. Gehrels and M. S. Matthews, eds., Arizona Univ. Press, Tucson, AZ, USA, 1989, 524-556; Buratti, B. J., Hillier, J. K. and Wang, M., The Lunar opposition surge: observations by Clemetine, Icarus, 1996, 124, 490-499; Goguen, J. D., A quantitative test of the applicability of independent scattering to high albedo planetary regoliths, 1999, Icarus, submitted; Helfenstein, P., Currier, N., Vlark, B. E., et al., Galileo observations of Europa's opposition effect, Icarus, 1998, 135, 41-63; Verbiscer, A. and Helfenstein, P., Reflectance spectroscopy of icy surfaces. In Solar System Ices, ed. B. Schmitt et al., Kluwer, Dordrecht, 1998, 157-197.

2. Brandt, R. E., Grenfell, T. C. and Warren, S. G., Optical properties of snow, Antarctic J. of the U.S., 1991, 26, 272-275.

3. Bréon, F.-M., Tanré, D., Lecomte, P. and Herman, M., Polarized reflectance of bare soils and vegetation: Measurements and models, IEEE Trans. Geosci. Remote Sens., 1995, 33, 487499.

4. Deering, D. W., Eck, T. F. and Otterman, J., Bidirectional reflectances of selected desert surfaces and their three-parameter soil characterization, Agricult. Forest Meteorol., 1990, 52 , $71-93$.

5. de Haan, J. F., Effects of aerosols on the brightness and polarization of cloudless planetary atmospheres, Ph.D. thesis, Free University, Amsterdam, 1987.

6. den Outer, P., Multiple light scattering in random discrete media: Coherent backscattering and imaging, Ph.D. thesis, University of Amsterdam, 1995.

7. Douté. S. and Schmitt, B., A multilayer bidirectional reflectance model for the analysis of planetary surface hyperspectral images at visible and near-infrared wavelengths, $J$. Geophys. Res., 1998, 103, 31,367-31,389. 
8. Dozier, J., Remote sensing of snow in the visible and near-infrared wavelengths. In Theory and Applications of Optical Remote Sensing, G. Asrar, ed.. Wiley, New York, 1989, $527-$ 547.

9. Fily, M., Bourdells, B., Dedieu, J. P. and Sergent, C., Comparison of in situ and Landsat Thematic Mapper derived snow grain characteristics in the Alps, Remote Sens. Environ., 1997, 59, 452-460; Fily, M., Leroux, C., Lenoble, J. and Sergent, C., Terrestrial snow studies from remote sensing in the solar spectrum and the thermal infrared. In Solar System Ices, ed. B. Schmitt et al., Kluwer, Dordrecht, 1998, 421-441.

10. Jin, Z. and Simpson, J. J., Bidirectional anisotropic reflectance of snow and sea ice in AVHRR channel 1 and 2 spectral regions-Part I: Theoretical analysis, IEEE Trans. Geosci. Remote Sens., 1999, 37, 543-554.

11. Gibbs, D. P., Betty, C. L., Fung, A. K., Blanchard, A. J., Irons, J. R. and Balsam, W. L., Automated measurement of polarized bidirectional reflectance, Remote Sens. Environ., 1993, $43,97-114$.

12. Grenfell, T. C., Warren, S. G. and Mullen, P. C., Reflection of solar radiation by the Antarctic snow surface at ultraviolet, visible, and near-infrared wavelengths, J. Geophys. Res., 1994, 99, 18,669-18,684.

13. Hapke, B., Theory of Reflectance and Emittance Spectroscopy, Cambridge Univ. Press, New York, 1993.

14. Hendricks, T. J. and Howell, J. R., Absorption/scattering coefficients and scattering phase functions in reticulated porous ceramics, ASME J. Heat Transfer, 1996, 118, 79-87.

15. Irons, J. R., Weismiller, R. A. and Petersen, G. W., Soil reflectance. In Theory and Applications of Optical Remote Sensing, G. Asrar, ed., Wiley, New York, 66-106.

16. Irons, J. R., Campbell, G. S., Norman, J. M., Graham, D. W. and Kovalick, W. M., Prediction and measurement of soil bidirectional reflectance, IEEE Trans. Geosci. Remote Sens., 1992, 30, 249-260.

17. Jafolla, J. C., Stokes, J. A. and Sullivan, R. J., Phenomenological BRDF modeling for engineering applications, Proc. SPIE, 1997, 3141, 1-12. 
18. Kuhn, M., Bidirectional reflectance of polar and alpine snow surfaces, Ann. Glaciol, 1985, 6, $164-167$.

19. Leroux, C. and Fily, M., Modeling the effect of sastrugi on snow reflectance, J. Geophys. Res., 1998, 103, 25,779-25,788.

20. Leroux, C., Deuzé, J.-L., Goloub, P., Sergent, C. and Fily, M., Ground measurements of the polarized reflectance of snow in the near-infrared spectral domain: Comparisons with model results, J. Geophys. Res., 1998, 103, 19,721-19,731.

21. Leroux, C., Lenoble, J., Brogniez, G., Hovenier, J. W. and de Haan, J. F., A model for the bidirectional polarized reflectance of snow, J. Quant. Spectrosc. Radiat. Transfer, 1999, 61, $273-285$.

22. Li, S., A model for the anisotropic reflectance of pure snow, Ph.D. thesis, University of California at Santa Barbara, USA, 1982.

23. Liang, S. and Mishchenko, M. I., Calculations of the hot-spot effect using the coherent backscattering theory, Remote Sens. Environ., 1997, 60, 163-173.

24. Lumme, K., Peltoniemi, J. I. and Irvine, W. M., Diffuse reflection from a stochastically bounded, semi-infinite medium, Transp. Theory Statist. Phys., 1990, 19, 317-332.

25. Martonchik, J. V., Diner, D. J., Pinty, B., Verstraete, M. M., Myneni, R. B., Knyazikhin, Yu. And Gordon, H. R., Determination of land and ocean reflective, radiative, and biophysical properties using multiangle imaging, IEEE Trans. Geosci. Remote Sens., 1998, 36, 12661281.

26. Mishchenko, M. I., The angular width of the coherent back-scatter opposition effect: An application to icy outer planet satellites, Astrophys. Space Sci., 1992, 194, 327-333.

27. Mishchenko, M. I., On the nature of the polarization opposition effect exhibited by Saturn's rings, Astrophys. J., 1993, 411, 351-361.

28. Mishchenko, M. I., Asymmetry parameters of the phase function for densely packed scattering grains, J. Quant. Spectrosc. Radiat. Transfer, 1994, 52, 95-110.

29. Mishchenko, M. I. and Dlugach, J. M., Can weak localization of photons explain the opposition effect of Saturn's rings? Mon. Not. Roy. Astron. Soc., 1992. 254, 15p-18p. 
30. Mishchenko, M. I. and Dlugach, J. M., Coherent backscatter and the opposition effect for Etype asteroids, Planet. Space Sci., 1993, 41, 173-181.

31. Mishchenko, M. I. and Macke, A., Asymmetry parameters of the phase function for isolated and densely packed spherical particles with multiple internal inclusions in the geometric optics limit, J. Quant. Spectrosc. Radiat. Transfer, 1997, 57, 767-794.

32. Muinonen, K., Coherent backscattering by solar system dust particles. In Asteroids, Comets, Meteors, A. Milani et al., eds., Kluwer, Dordrecht, 1993, 271-296.

33. Nelson, H. F., Look, D. C. and Crosbie, A. L., Two-dimensional radiative back-scattering from optically thick media, ASME J. Heat Transfer, 1986, 108, 619-625.

34. Nolin, A. W. and Dozier, J., Estimating snow grain size using AVIRIS data, Remote Sens. Environ., 1993, 44, 231-238.

35. Peltoniemi, J. I. and Lumme, K., Light scattering by closely packed particulate media, J. Opt. Soc. Am. A, 1992, 9, 1320-1326.

36. Pinty, B. and Verstraete, M. M., On the design and validation of surface bidirectional reflectance and albedo models, Remote Sens. Environ., 1992, 41, 155-167.

37. Pitter, M. C., Jakeman, E. and Harris, M., Coherent detection of enhanced back-scatter from rough surfaces and particle suspensions, $J$. Mod. Opt., 1998, 45, 1557-1565.

38. POAN Research Group, ed., New Aspects of Electromagnetic and Acoustic Wave Diffusion, Springer, Berlin, 1998.

39. Steffen, K., Effect of solar zenith angle on snow anisotropic reflectance. In IRS'96: Current Problems in Atmospheric Radiation, W. L. Smith and K. Stamnes, eds., Deepak Publ., Hampton, VA, USA, 1997, 41-44.

40. Taylor, V. R. and Stowe, L., Reflectance characteristics of uniform earth and cloud surfaces derived from NIMBUS-7 ERB, J. Geophys. Res., 1984, 89, 4987-4996.

4L. Tien, C. L., Thermal radiation in packed and fluidized beds, ASMIE J. Heat Transfer, 1988, $110,1230-1242$.

42. van Der Mark, M. B., Propagation of light in disordered media: A search for Anderson Localization, Ph.D. thesis. University of Amsterdam, 1990. 
43. van Tiggelen, B. A., Multiple scattering and localization of light, Ph.D. thesis, University of Amsterdam, 1992.

44. Tsay, S.-C., King, M. D., Arnold, G. T. and Li, J. Y., Airborne spectral measurements of surface anisotropy during SCAR-B, J. Geophys. Res., 1998, 103, 31,943-31,953; Soulen, P. F., Tsay, S.-C., King, M. D., Arnold, G. T. and Jason, Y. L., Airborne spectral measurements of surface anisotropy during the SCAR-A, Kuwait oil fire, and TARFOX experiments, $J$. Geophys. Res., 1999, in press.

45. Warren, S. G., Optical properties of snow, Rev. Geophys., 1982, 20, 67-89.

46. Warren, S. G., Brandt, R. E. and Hinton, P. O'R., Effect of surface roughness on bidirectional reflectance of Antarctic snow, J. Geophys. Res., 1998, 103, 25,789-25,807.

47. Hansen, J. E., and Travis, L. D., Light scattering in planetary atmospheres, Space Sci. Rev., $1974,16,527-610$.

48. de Haan, J. F., Bosma, P. B. and Hovenier, J. W., The adding method for multiple scattering calculations of polarized light, Astron. Astrophys., 1987, 183, 371-391.

49. Stamnes, K., The theory of multiple scattering of radiation in plane parallel atmospheres, Rev. Geophys., 1986, 24, 299-310.

50. Sobolev, V. V., Light Scattering in Planetary Atmospheres, Pergamon Press, Oxford, 1975, Chapter 2.

51. Dlugach, J. M and Yanovitskij, E. G., The optical properties of Venus and the Jovian planets. II. Methods and results of calculations of the intensity and radiation diffusely reflected from semi-infinite homogeneous atmospheres, Icarus, 1974, 22, 66-81; Yanovitskij, E. G., Light Scattering in Inhomogeneous .Atmospheres, Springer, Berlin, 1997, Chapters 2 and 3.

52. de Rooij, W. A., Reflection and transmission of polarized light by planetary atmospheres, Ph.D. thesis, Free University, Amsterdam, 1985.

53. Mishchenko, M. I., Lacis, A. A. and Travis, L. D., Errors induced by the neglect of polarization in radiance calculations for Rayleigh-scattering atmospheres, J. Quant. Spectrosc. Radiat. Transfer, 1994, 51, 491-510. 
54. Lacis, A. A., Chowdhary, J., Mishchenko, M. I. and Cairns, B., Modeling errors in diffusesky radiation: Vector vs. scalar treatment, Geophys. Res. Lett., 1998, 25, 135138.

55. Hansen, J. E.. Multiple scattering of polarized light in planetary atmospheres. II. Sunlight reflected by terrestrial water clouds, J. Atmos. Sci., 1971, 28, 1400-1428.

56. van de Hulst, H. C., Multiple Light Scattering, Academic Press, New York, 1980.

57. Gelfand, I. M., Minlos, R. A. and Shapiro, Z. Ya., Representations of the Rotation and Lorentz Groups and Their Applications. Pergamon Press, Oxford, 1963.

58. Hovenier, J. W. and van der Mee, C. V. M., Fundamental relationships relevant to the transfer of polarized light in a scattering atmosphere, Astron. Astrophys., 1983, 128, 1-16.

59. de Rooij, W. A. and Domke, H., On the nonuniqueness of solutions for nonlinear integral equations in radiative transfer theory, J. Quant. Spectrosc. Radiat. Transfer, 1984, 31, 285-299.

60. Irvine, W. M., Multiple scattering by large particles, Astrophys. J., 1965, 142, 1563-1575.

61. King, M. D., Number of terms required in the Fourier expansion of the reflection function for optically thick atmospheres, J. Quant. Spectrosc. Radiat. Transfer, 1983, 30, 143-161.

62. de Rooij, W. A. and van der Stap, C. C. A. H., Expansion of Mie scattering matrices in generalized spherical functions, Astron. Astrophys., 1984, 131, 237-248.

63. Lacis, A. A. and Mishchenko, M. I., Climate forcing, climate sensitivity, and climate response: A radiative modeling perspective on atmospheric aerosols. In Aerosol Forcing of Climate, R. J. Charlson and J. Heintzenberg, eds., Wiley, New York, 1995, 11-42.

64. Mishchenko, M. I. and Travis, L. D., Capabilities and limitations of a current FORTRAN implementation of the $T$-matrix method for randomly oriented, rotationally symmetric scatterers, J. Quant. Spectrosc. Radiat. Transfer, 1998, 60, 309-324.

65. Mishchenko, M. I. Travis, L. D. and Mackowski, D. W., T-matrix computations of light scattering by nonspherical particles: A review, J. Quant. Spectrosc. Radiat. Transfer, 1996, $55,535-575$.

66. Mishchenko, M. I., Light scattering by randomly oriented axially symmetric particles, J. Opt. Soc. Am. A, 1991, 8, 871-882. 
67. Muinonen, K., Lumme, K., Peltoniemi, J. and Irvine, W. M., Light scattering by randomly oriented crystals, Appl. Opt., 1989, 28, 3051-3060.

68. Takano, Y. and Liou, K. N., Solar radiative transfer in cirrus clouds. I: Single-scattering and optical properties of hexagonal ice crystals, J. Atmos. Sci., 1989, 46, 3-19.

69. Mishchenko, M. I. and Macke, A., Incorporation of physical optics effects and computation of the Legendre expansion for ray-tracing phase functions involving $\delta$-function transmission, $J$. Geophys. Res., 1998, 103, 1799-1805.

70. Dave, J. V. and Armstrong, B. H., Computation of high-order associated Legendre polynomials, J. Quant. Spectrosc. Radiat. Transfer, 1970, 10, 557-562.

71. Krylov, V. I., Approximate Calculation of Integrals, Macmillan, New York, 1962.

72. Adams, C. N. and Kattawar, G. W., Solutions of the equations of radiative transfer by an invariant imbedding approach, J. Quant. Spectrosc. Radiat. Transfer, 1970, 10, 341-356.

73. Lenoble, J., ed., Radiative Transfer in Scattering and Absorbing Atmospheres: Standard Computationsl Procedures. Deepak, Hampton, VA, USA, 1985.

74. Nakajima, T. and King, M. D., Asymptotic theory for optically thick layers: application of the discrete ordinates method, Appl. Opt., 1992, 31, 7669-7683.

75. Hansen, J. E. and Pollack, J. B., Near-infrared light scattering by terrestrial clouds, J. Atmos. Sci., 1970, 27, 265-281.

76. Ishida, T., Ando, H. and Fukuhara, M., Estimation of complex refractive index of soil particles and its dependence on soil chemical properties, Remote Sens. Environ., 1991, 38, $173-182$.

77. Jaggard, D. L., Hill, C., Shorthill, R. W. et al.. Light scattering from particles of regular and irregular shape, Atmos. Environ., 1991, 15, 2511-2519.

78. Mishchenko, M. I., Travis, L. D., Kahn, R. A. and West, R. A., Modeling phase functions for dustlike tropospheric aerosols using a shape mixture of randomly oriented polydisperse spheroids, J. Geophys. Res., 1997, 102, 16,831-16,847. 
79. West, R. A., Doose, L. R., Eibl, A. M. et al., Laboratory measurements of mineral dust scattering phase function and linear polarization, J. Geophys. Res., 1997, 102, 16,871-16,881.

80. Muñoz, O., Volten, H., Rol, E. et al., Experimental determination of scattering matrices of mineral particles. In Light Scattering by Nonspherical Particles: Theory, Measurements, and Applications, M. I. Mishchenko, L. D. Travis and J. W. Hovenier, eds., American Meteorological Society, Boston, MA, USA, 1998, 265-268.

81. Hovenier, J. W. and Hage, J. I., Relations involving the spherical albedo and other photometric quantities of planets with thick atmospheres, Astron. Astrophys., 1989, 214, 391-401.

82. Joseph, J. H., Wiscombe, W. J. and Weinman, J. A., The delta-Eddington approximation for radiative flux transfer, J. Atmos. Sci., 1976, 33, 2452-2459.

83. Macke, A., Mueller, J. and Raschke, E., Scattering properties of atmospheric ice crystals, $J$. Atmos. Sci., 1996, 53, 2813-2825.

84. Mishchenko, M. I., Rossow, W. B., Macke, A. and Lacis, A. A., Sensitivity of cirrus cloud albedo, bidirectional reflectance and optical thickness retrieval accuracy to ice particle shape, $J$. Geophys. Res., 1996, 101, 16,973-16,985.

85. Kokhanovsky, A., Optics of Light Scattering Media: Problems and Solutions, Wiley-Praxis, Chichester, 1999.

86. Bohren, C. F. and Beschta, R. L., Snowpack albedo and snow density, Cold Reg. Sci. Technol., 1979, 1, 47-50.

87. Wiscombe, W. J. and Warren, S. G., A model for the spectral albedo of snow. I: Pure snow, J. Atmos. Sci., 1980, 37, 2712-2733.

88. Fraden, S. and Maret, G., Multiple light scattering from concentrated, interacting suspensions, Phys. Rev. Lett., 1990, 65, 512-515.

89. Kumar, S. and Tien, C. L., Dependent absorption and extinction of radiation by small particles, ASME J. Heat Transfer, 1990, 112, 178-185.

90. Göbel, G., Kuhn, J. and Fricke, J., 1995, Dependent scattering effects in latex-sphere suspensions and scattering powders, Waves Random Media, 1995, 5, 413-426. 
91. Shinde, R., Balgi, G., Richter, S., Banerjee, S., Reynolds, J., Pierce. J. and Sevick-Muraca, E., Investigation of static structure factor in dense suspensions by use of multiply scattered light, Appl. Opt., 1999, 38, 197-204.

92. Barabanenkov, Yu. N., Kravtsov, Yu. A., Ozrin, V. D. and Saichev, A. I., Enhanced backscattering in optics. In Progress in Optics, E. Wolf, ed., Vol. XXIX, Elsevier, Amsterdam, $1991,65-197$.

93. Mishchenko, M. I., Diffuse and coherent backscattering by discrete random media. I. Radar reflectivity, polarization ratios, and enhancement factors for a half-space of polydisperse, nonabsorbing and absorbing spherical particles, J. Quant. Spectrosc. Radiat. Transfer, 1996, 56, 673-702.

94. Fung, A. K. and Eom, H. J., A study of backscattering and emission from closely packed inhomogeneous media, IEEE Trans. Geosci. Remote Sens., 1985, 23, 761-767.

95. Chuah, H.-T., Tjuatja, S., Fung, A. K. and Bredow, J. W., A phase matrix for a dense discrete random medium: evaluation of volume scattering coefficient, IEEE Trans. Geosci. Remote Sens., 1996, 34, 1137-1143.

96. Shepard, M. K. and Campbell, B. A., Shadows on a planetary surface and implications for photometric roughness, Icarus, 1998, 134, 279-291.

97. Hillier, J. K., Scattering of light by composite particles in a planetary surface, Icarus, 1997, $130,328-335$. 


\section{FIGURE CAPTION}

Fig. 1. Spherical coordinates specifying the direction of light propagation. The zenith and azimuth angles of the incident beam are $\vartheta_{0}>\pi / 2$ and $\varphi_{0}=0$, respectively.

Fig. 2. Phase functions for soil particle models $1-4$ (upper panel) and snow particle models 1-3. Dotted curves in the upper panel show asymmetry-parameter-equivalent Henyey-Greenstein phase functions.

Fig. 3. Upper panel: plane albedo versus $\mu_{0}$ for soil particle models $1-4$. Lower panel: plane albedos computed using the $\delta$-Eddington approximation (solid curves) and asymmetryparameter-equivalent Henyey-Greenstein phase functions (dotted curves) relative to the exact values for soil particle models $1-4$.

Fig. 4. Left column: reflected intensity versus $\mu$ and $\varphi$ for soil particle model 1. Middle column: the same but for the asymmetry-parameter-equivalent Henyey-Greenstein phase function. Right column: the ratio of the intensities shown in the middle and left columns. The four values of the cosine of the illumination zenith angle $\mu_{0}=0.9,0.7,0.4$, and 0.1 are indicated by the yellow stars in the right column. The azimuth angle of the incident radiation is zero.

Fig. 5. Reflected intensity versus $\mu$ and $\varphi$ for snow particle models 1,2 , and 3 . The four values of the cosine of the illumination zenith angle $\mu_{0}=0.9,0.7,0.4$, and 0.1 are indicated by the yellow stars. The azimuth angle of the incident radiation is zero.

Fig. 6. As in Plate 4, but for ratios of reflected intensities. 
Table 1. Division points and weights of the Markov quadrature formula on the interval $[0,1]$ with $n=30$

\begin{tabular}{|c|c|c|}
\hline & $\mu$ & $w$ \\
\hline 1 & 0.00160587785254 & $0.00+11899+13797$ \\
\hline 2 & $0.008+4194880403$ & 0.00954444032329 \\
\hline 3 & 0.02066193363616 & 0.01487343889309 \\
\hline 4 & 0.03813469699711 & 0.02004018939073 \\
\hline 5 & 0.06066914207658 & 0.02498751843953 \\
\hline 6 & 0.08801845813989 & 0.02966112217483 \\
\hline 7 & 0.11988302768490 & 0.03400976899322 \\
\hline 8 & 0.15591374708438 & 0.03798580509944 \\
\hline 9 & 0.19571586157012 & 0.04154566451353 \\
\hline 10 & 0.23885329363337 & 0.04465034297146 \\
\hline 11 & 0.28485342210352 & 0.04726582410455 \\
\hline 12 & 0.33321226086401 & 0.04936345167913 \\
\hline 13 & 0.38339998090611 & 0.05092024336491 \\
\hline 14 & 0.43486671538603 & 0.05191914244132 \\
\hline 15 & 0.48704858415304 & 0.05234920462588 \\
\hline 16 & 0.53937387177272 & 0.05220571795295 \\
\hline 17 & 0.59126929137078 & 0.05149025437772 \\
\hline 18 & 0.64216626567319 & 0.05021065253237 \\
\hline 19 & 0.69150715642309 & 0.04838093181641 \\
\hline 20 & 0.73875137391194 & 0.04602113875159 \\
\hline 21 & 0.78338129966059 & 0.04315712726339 \\
\hline 22 & 0.82490795730055 & 0.03982027524838 \\
\hline 23 & 0.86287636938624 & 0.03604714041164 \\
\hline 24 & 0.89687054110030 & 0.03187905880664 \\
\hline 25 & 0.92651801527194 & 0.02736168939988 \\
\hline 26 & 0.95149394561648 & 0.02254450560005 \\
\hline 27 & 0.97152463085599 & 0.01748022010489 \\
\hline 28 & 0.98639040253929 & 0.01222402012888 \\
\hline 29 & 0.99592721636071 & 0.00683100534121 \\
\hline 30 & 1.00000000000000 & 0.00111111111111 \\
\hline
\end{tabular}


Table 2. Soil particle models

\begin{tabular}{|c|c|c|c|c|c|c|c|c|c|c|}
\hline Model & $a(\mathrm{~m} \mu)$ & $b$ & $m_{r}$ & $m_{i}$ & $\varpi$ & $<\cos \theta>$ & $s_{\max }$ & $d_{S}$ & $A_{S}(H G)$ & $d_{J}(\mathrm{HH})$ \\
\hline 1 & 10 & 0.1 & 1.55 & 0.001 & 0.85404 & 0.83752 & $6+1$ & 0.1399 & 0.1382 & 0.1655 \\
\hline 2 & 10 & 0.1 & 1.55 & 0.002 & 0.76137 & 0.86568 & $6+4$ & 0.0727 & 0.0716 & 0.0889 \\
\hline 3 & 10 & 0.1 & 1.55 & 0.003 & 0.69923 & 0.88582 & $6+5$ & 0.0472 & 0.0464 & 0.0588 \\
\hline 4 & 10 & 0.1 & 1.55 & 0.004 & 0.65646 & 0.90054 & 646 & 0.0345 & 0.0339 & 0.0435 \\
\hline
\end{tabular}

Table 3. Snow particle models

\begin{tabular}{ccccccccc}
\hline Model & Shape & $r_{\text {eff }}(\mu \mathrm{m})$ & $v_{\text {eff }}$ & $m_{r}$ & $m_{i}$ & $\varpi$ & $<\cos \Theta>$ & $s_{\max }$ \\
\hline & & & & & & & & \\
1 & Irregular & 50 & 0.2 & 1.311 & 0 & 1 & 0.7524 & 2000 \\
2 & Spherical & 50 & 0.2 & 1.311 & 0 & 1 & 0.8860 & 1948 \\
3 & Hexagonal & 50 & 0.2 & 1.311 & 0 & 1 & 0.8117 & 2000 \\
\hline
\end{tabular}




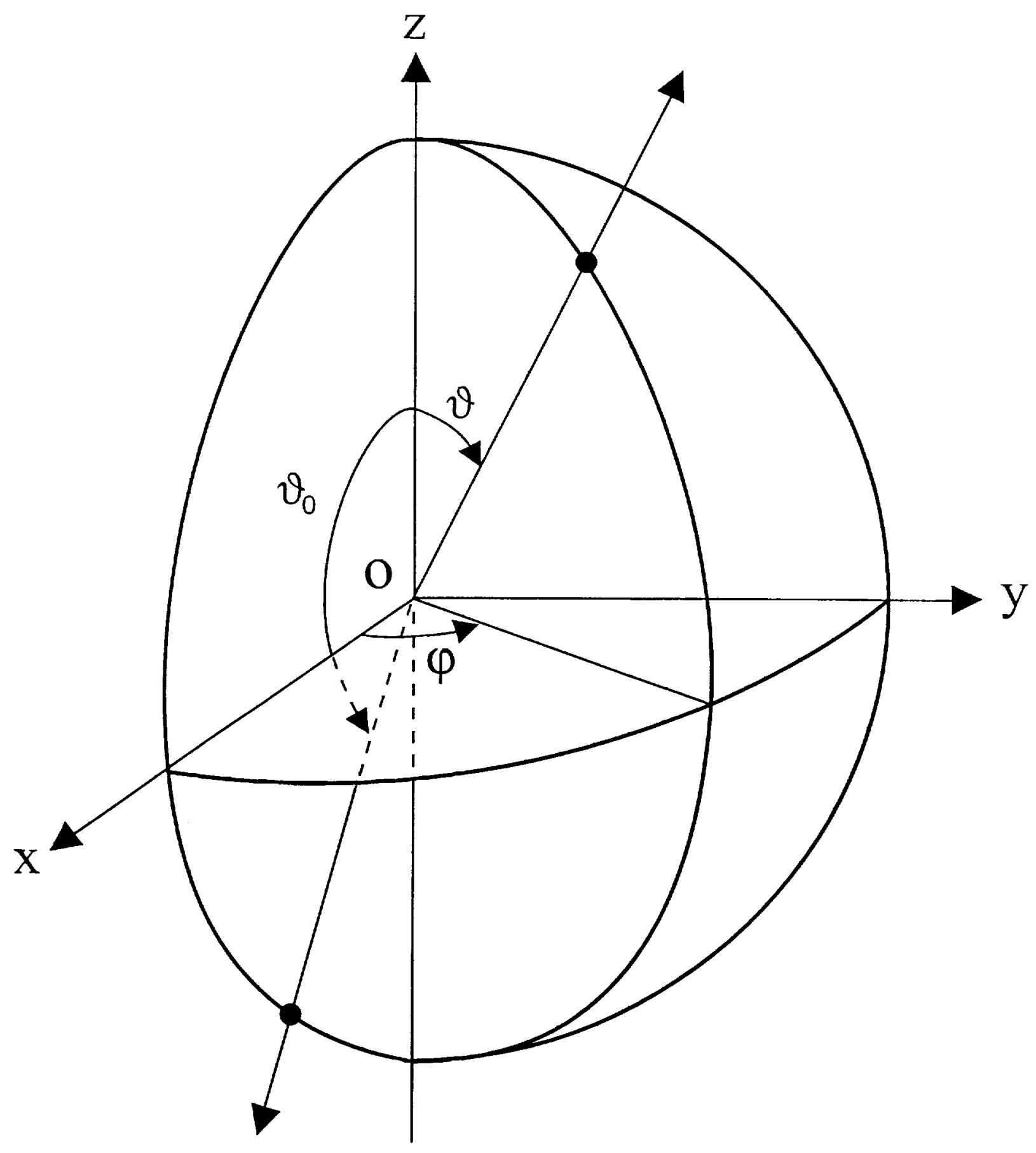

Fig. I 


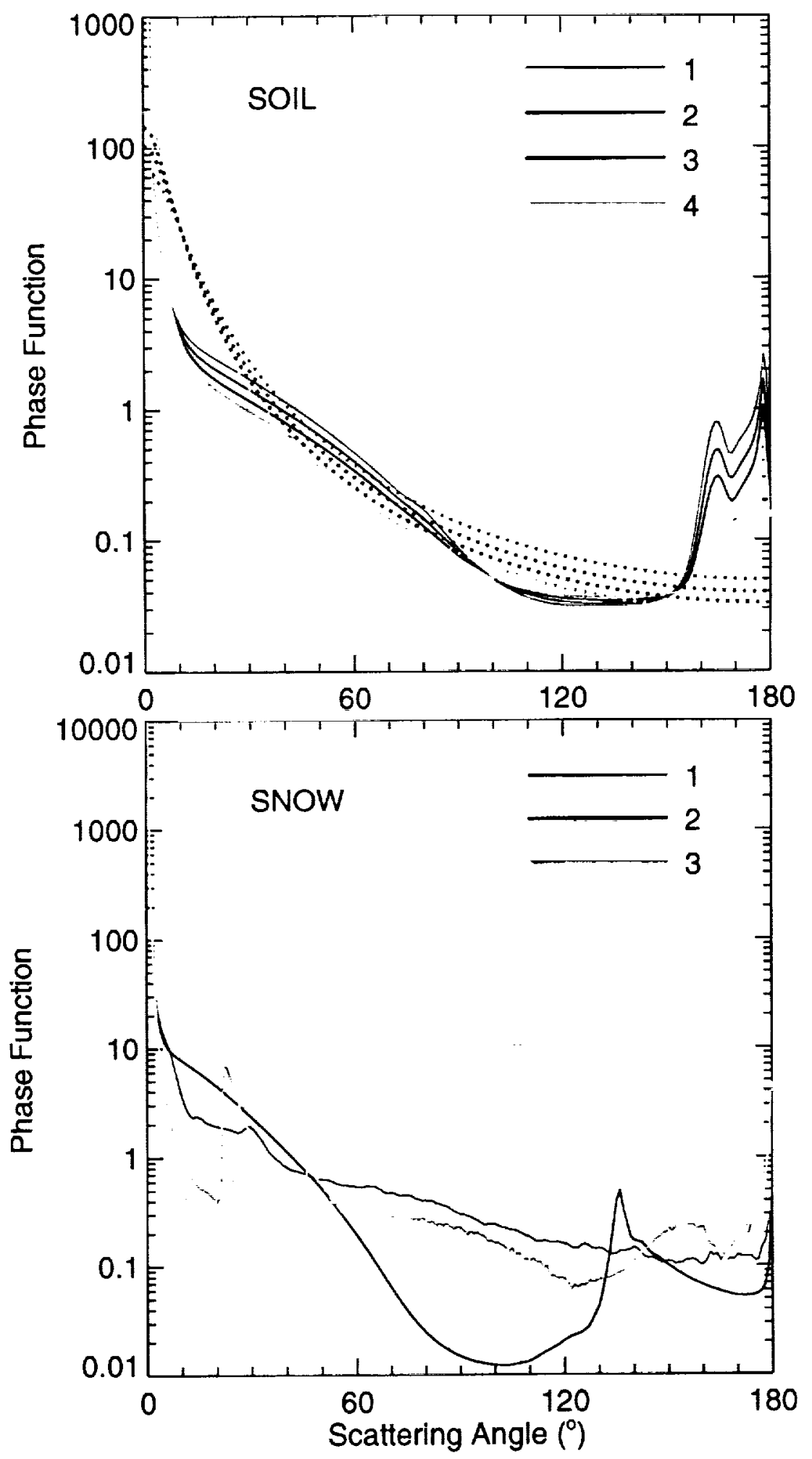




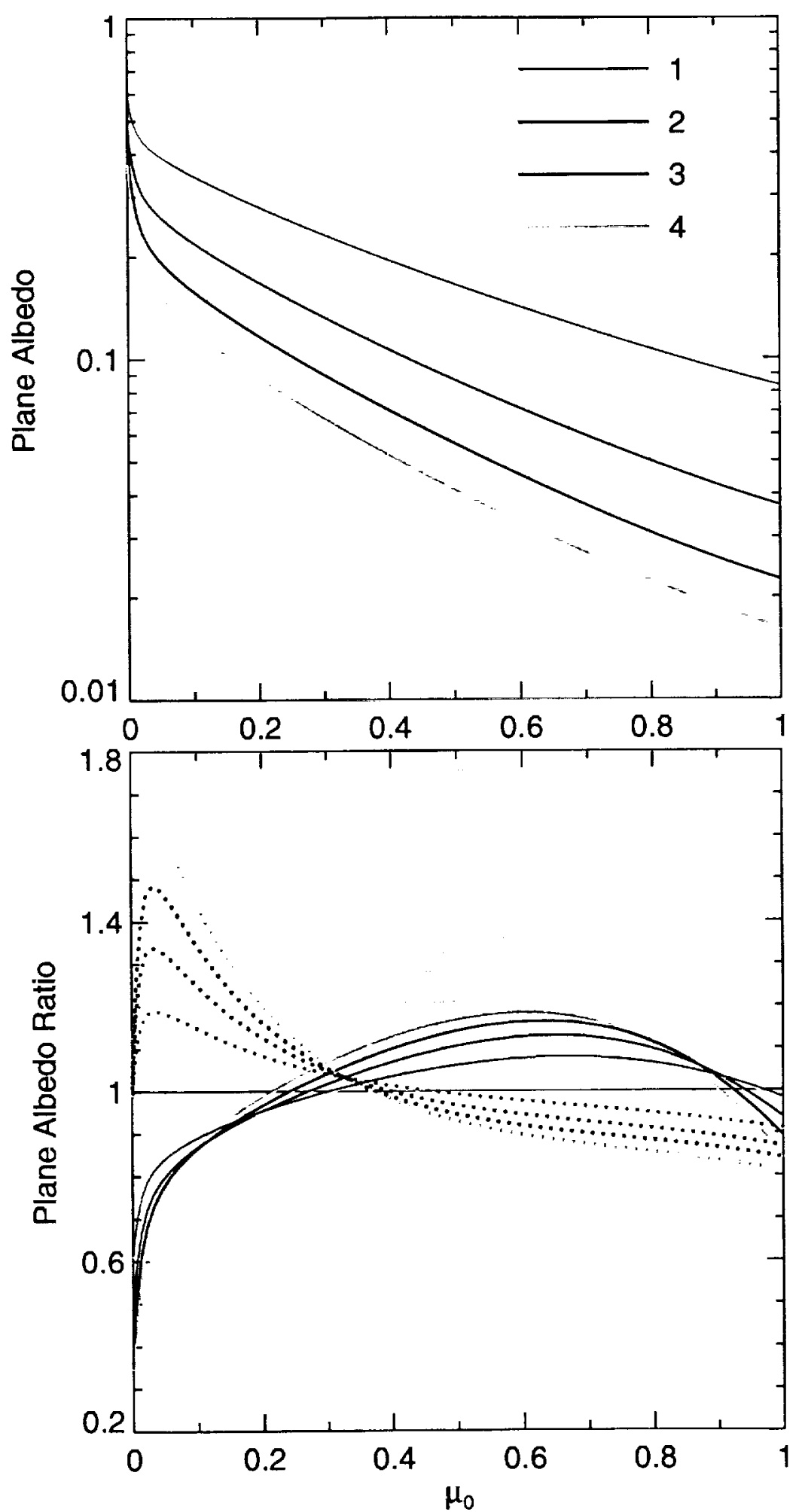



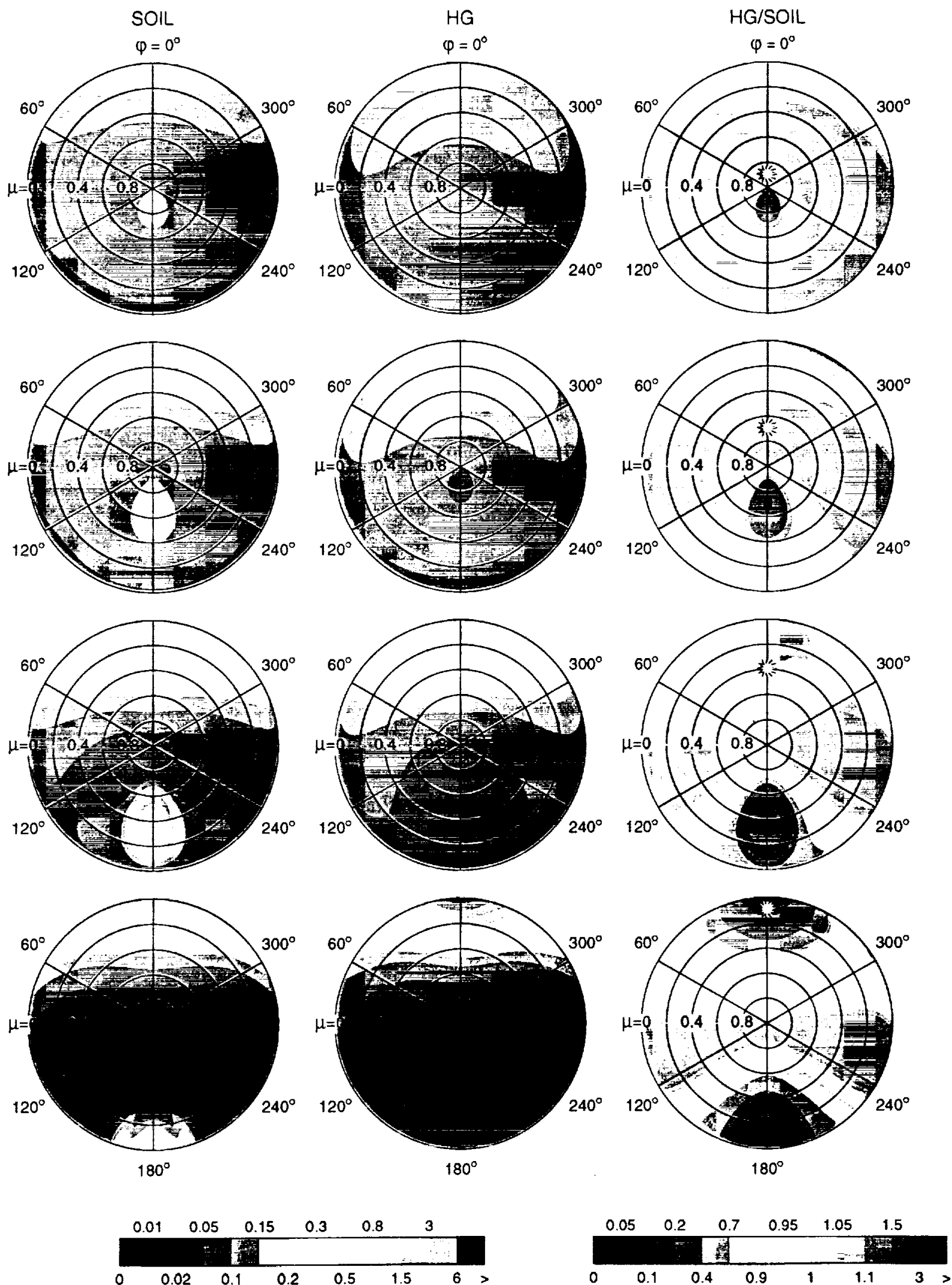

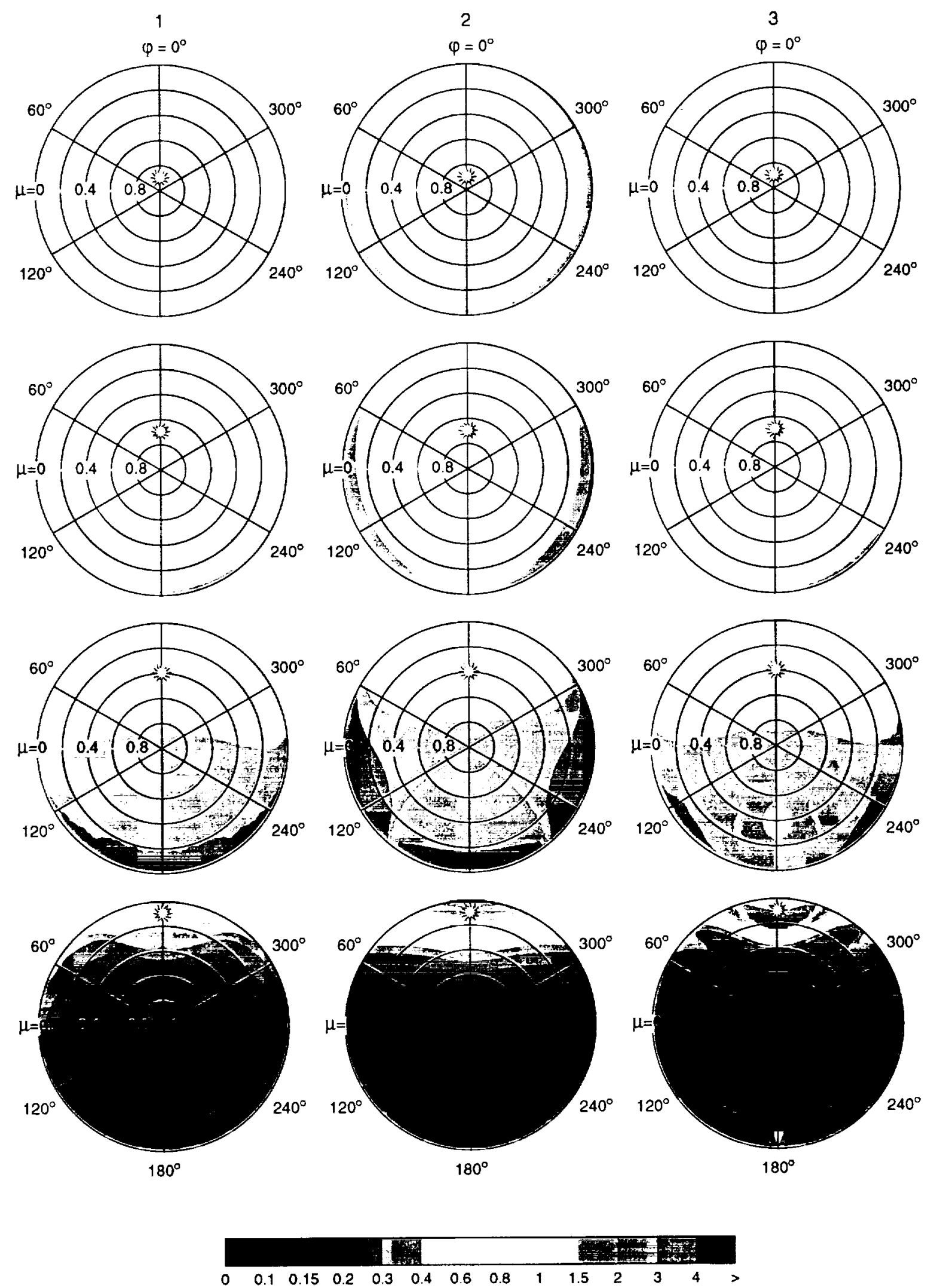

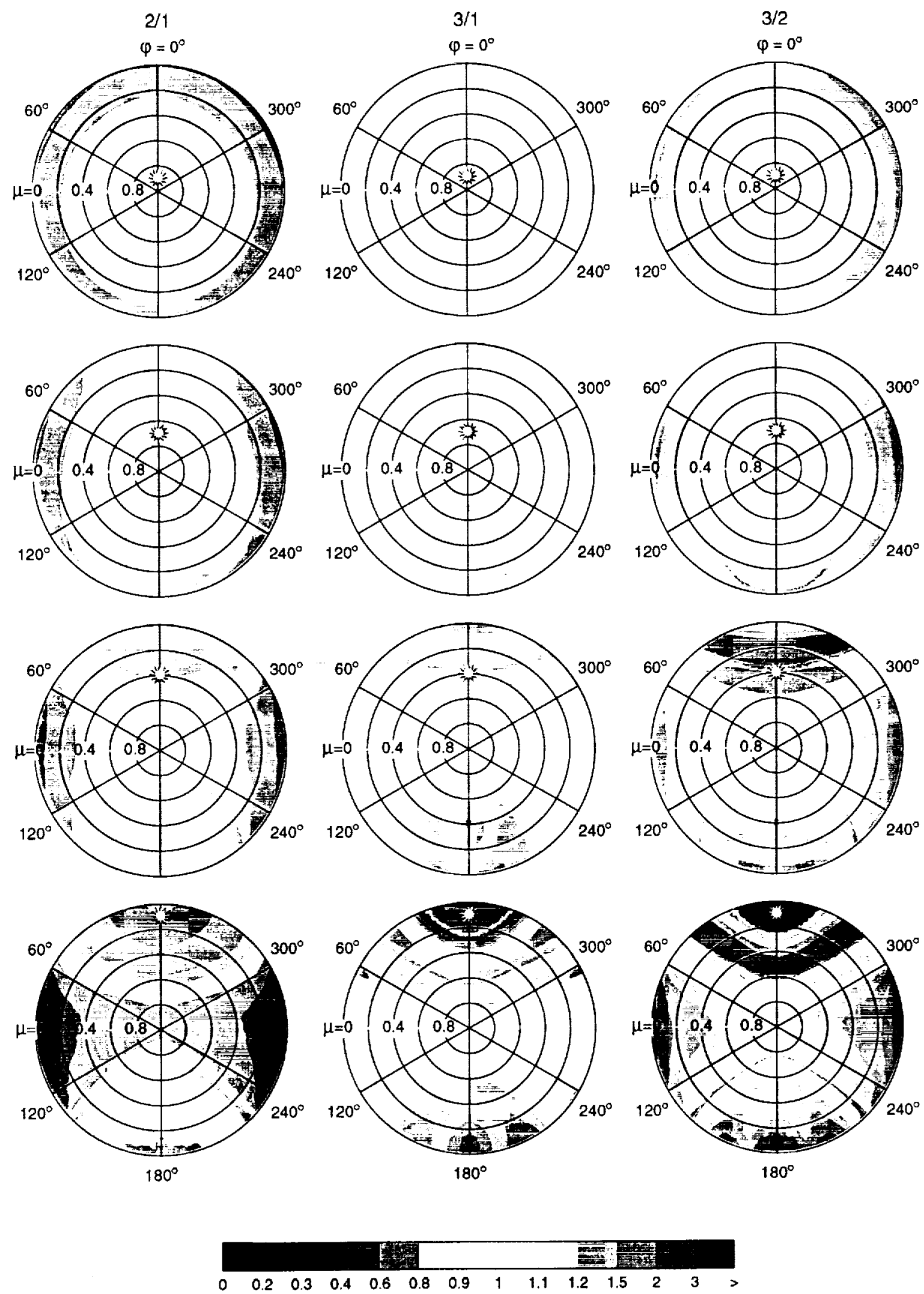